\title{
A long and winding sTORy
}

\author{
This year's Albert Lasker Basic Medical Research Award honoured Michael Hall for his work on the TOR proteins. \\ We congratulate the new Lasker laureate and take this opportunity to revisit the history of this fundamental \\ signalling pathway.
}

The story of the TOR (target of rapamycin) pathway starts in a location much more exotic than a research lab, with an expedition to the Easter Island, also known as Rapa Nui, in the 1960s. Soil samples collected during this venture eventually led to the isolation from the bacterium Streptomyces hygroscopicus of a macrolide with antifungal activity, which was aptly named rapamycin. Although this compound was found to also have antiproliferative and immunosuppressive activity, its mechanism of action remained a mystery until the early 1990s when Michael Hall and co-workers at the Biozentrum of the University of Basel set up genetic screens to identify genes that when mutated would permit Saccharomyces cerevisiae yeast cells to escape the drug's cytostatic effects. This led to the seminal discovery of two related genes, named TOR1 and TOR2 (Science 253, 905-909; 1991), which were later shown to have kinase activity and to be required for cell proliferation (Cell 73, 585-596; 1993). Soon after Michael Hall's discovery of the yeast TOR proteins, their mammalian counterparts were independently identified by Stuart Schreiber (Nature 369, 756-758; 1994), David Sabatini and Solomon Snyder (Cell 78, 35-43; 1994), Vivian Berlin (Proc. Natl Acad. Sci. USA 91, 12574-12578; 1994) and Robert Abraham (J. Biol. Chem. 270, 815-822; 1995). Although at the time the mammalian orthologue was given different names by the different groups reporting its identification, the name mammalian TOR, or mTOR, quickly prevailed in the community, and with modification 'mechanistic TOR' or MTOR was later adopted by the HUGO gene nomenclature committee as the official name.

These early findings were followed by the identification of upstream regulators and downstream effectors of $\mathrm{mTOR}$, and a new signalling pathway started to emerge (reviewed in Cell 168, 960-976; 2017). By the end of the 1990s, mTOR was shown to phosphorylate two major substrates involved in translation, the S6K1 kinase and 4EBP1, followed by a series of key publications from multiple laboratories in this and other journals that shaped the core pathway in the early 2000s. Work in Drosophila and mammalian cells demonstrated that the TSC1 and TSC2 tumour suppressors inhibited mTOR activity by promoting GTP hydrolysis of the Rheb GTPase. At this time, growth factor signalling and the PI3K-AKT pathway were also placed upstream of mTOR by demonstrating that Akt phosphorylated and inhibited TSC2. mTOR itself was found to represent two separate complexes, mTOR complex 1 (mTORC1) and mTOR complex 2 (mTORC2), with only mTORC1 being rapamycin-sensitive. These two complexes were shown to have distinct downstream responses, with S6K1, 4EBP1 and several key transcription factors placed downstream of mTORC1, and kinases such as AKT, SGK and PKC downstream of mTORC2. mTORC1 and mTORC2 are also subject to distinct regulation: although both are controlled by growth factor signalling, mTORC1 additionally responds to changes in nutrients, amino acids, energy status and oxygen levels.
Moreover, elegant work in the last decade has added the amino-aciddependent regulatory network that controls the Rag GTPases to the inputs upstream of mTORC1 activity, and has established the lysosome as the key activation platform for mTORC1.

Research into the functional outcomes of mTOR has revealed a similar level of diversity (Cell 168, 960-976; 2017). Very early on, loss of mTOR activity was shown to prevent cell cycle progression, and this proliferative role of mTOR was soon linked to its control of anabolic cellular processes. For example, mTORC1 is known to promote mRNA translation, and lipid and nucleotide synthesis, but also inhibits catabolic processes such as autophagy. Separately, both mTORC1 and mTORC2 are involved in the control of glucose metabolism, and mTORC2 inhibits apoptosis but further regulates cytoskeletal remodelling and cell migration. This multitude of cellular functions translates to crucial roles in physiology and disease at the organismal level. mTOR signalling has notable roles in glucose homeostasis in the liver and pancreas, regulation of muscle mass, adipogenesis and lipid metabolism. As a fundamental pathway regulating cell growth and metabolic homeostasis, mTOR is unsurprisingly frequently activated in cancer. Substantial efforts to characterize its complex roles and dysregulation in this setting have led to some positive clinical results, with rapamycin analogues approved for treatment of a number of cancers, and ongoing work at the bench and in the clinic to identify other mTOR-targeting strategies. mTOR has also been implicated in organismal ageing with the underlying mechanisms being actively investigated.

For a cell biologist in 2017, it is hard to imagine the time when a pathway as central to cellular homeostasis as MTOR was still uncharted territory. Indeed, more than 25 years after its discovery, the textbook page of mTOR's regulation and functions is very heavily populated. Looking back, it is important to remember that this was the outcome of fundamental discoveries from multiple researchers, who conducted work in systems as diverse as yeast, mammalian cell lines, mice and Drosophila through a combination of genetics, biochemistry, molecular and cell biology. We congratulate Michael Hall for the Lasker Award and for seeding the discovery of TOR. We thank him and the many other researchers who contributed to the elucidation of the pathway, and apologize to all the scientists whose work we did not have space to acknowledge in this short piece.

mTOR has been and remains the source of surprises. During the signalling heyday of the 1990s, it provided the conceptual leap that cell growth is an actively regulated process. More recently, it revealed the lysosome as a platform for amino-acid-dependent signalling, and continues to be one of the most active areas of cell biological research. With that in mind we look forward to the new and exciting chapters awaiting to be written in the fascinating story of TOR. 